\title{
Miopatia do Exercício. Anatomopatologia e Fisiopatologia
}

\author{
J. A. Duarte \\ M.P. Mota \\ M.J. Neuparth \\ H.J. Appell \\ J.M.C. Soares
}

Faculdade de Ciências do Desporto e Educação Física

Universidade do Porto, Porto, Portugal

https://doi.org/10.5628/rpcd.01.02.73

\section{RESUMO}

O exercício físico exaustivo e inabitual induz alterações estruturais e ultra-estruturais musculares esqueléticas cuja

fisiopatologia é ainda pouco clara. Neste trabalho são descritas, em pormenor, essas alterações observadas após exercício, dando especial realce às irregularidades do padrão estriado, à palidez de coloração com o ácido periódico de Schiff, à vacuolização sarcoplasmática, às áreas de necrose segmentar, à presença de núcleos centrais, à activação das células satélite e fibroblastos, ao edema intra-celular e à reacção inflamatória muscular com infiltração de fagócitos. São ainda analisados os seus tempos de aparecimento e os hipotéticos mecanismos subjacentes. Na génese destas anomalias parecem estar factores de natureza metabólica e mecânica, sendo a participação de cada um condicionada pelo tipo de exercício efectuado. Tudo indica que o quadro anatomopatológico seja variável, com predominância de um ou outro tipo de alterações, em função do exercício realizado.

Palavras-chave: Miopatia do exercício, alterações estruturais musculares, fisiopatologia, factores condicionantes.

\author{
ABSTRACT \\ Exercise induced muscle damage. \\ Anatomopathology and Physiopathology.
}

Exhaustive and unusual physical exercise induces structural and ultra-structural alterations in skeletal muscular whose physiopathology is still unclear. These alterations are described in detail, giving special importance to irregularities of cross striated pattern, paleness with Schiff periodic acid, vacuolization, segmental necrosis, central nuclei, activation of satellite cells and fibroblasts, intra-cellular edema and to the muscular inflammatory reaction with phagocytes infiltration. Their onset times and hypothetical underlying mechanisms are also analyzed. In the origin of these anomalies are metabolic and mechanical factors whose contribution is conditioned by the type of exercise. Experimental data suggests that morphological characteristics of muscle damage induced by exercise are variable according to the metabolic or mechanical stress of exercise.

Key Words: Exercise induced muscle damages, structural damages, Physiopathology. 
A miopatia do exercício (MEx) é uma entidade anatomopatológica induzida pela realização de exercícios físicos extenuantes ou inabituais, limitada a algumas áreas do tecido muscular estriado esquelético, surgindo tanto em animais normais de laboratório como em humanos clinicamente saudáveis $(8,9,12,16,20,22)$. É identificada, no Homem e no modelo animal, pela elevação das enzimas lisossómicas musculares, pela elevação plasmática de várias proteínas musculares, pelas alterações estruturais e ultra-estruturais musculares e pela diminuição da força máxima voluntária e involuntária não atribuível à fadiga $(3,4,5,13,17$, 20). No Homem, a sensação retardada de desconforto muscular e a captação aumentada de produtos radioactivos pelos músculos lesados são também sinais clinicamente importantes $(6,7,13)$. Se o termo "miopatia" parece ser abusivo, o seu carácter necrótico, catabólico e inflamatório, apesar de transitório (7), justifica a designação. Da mesma forma, muitas das alterações funcionais e morfológicas musculares de origem endócrina, metabólica ou medicamentosa são também designadas por miopatias, apesar de transitórias e reversíveis (para refs. ver 7). Importa referir que alterações estruturais e ultra-estruturais semelhantes àquelas encontradas após exercício exaustivo e inabitual, são também observadas em doentes com poliomiosite e distrofia muscular. De facto, sendo muito limitado o número de reacções básicas das fibras musculares aos vários estímulos agressivos, é natural que diferentes agressões possam induzir a mesma reacção celular $(1,2,10$, 11, 21). Assim, não é de estranhar que as lesões descritas para a MEx não sejam específicas desta, sendo, por isso, encontradas em outras patologias musculares.

Em contraste com as lesões agudas motivadas pela prática desportiva, tais como as rupturas musculares, este estado patológico do músculo esquelético é caracterizado por possuir uma natureza retardada $(3,4,5,7,17)$. De facto, as alterações estruturais associadas à miopatia do exercício são bem diferentes daquelas induzidas por exercícios físicos violentos. Essas traduzem-se, habitualmente, por dilaceração e/ou ruptura de pequenas veias e das fibras musculares, com possível formação de hematomas, de aparecimento súbito, sendo vulgarmente causadas por contusões externas ou pela aplicação de forças musculares excessivas. De acordo com os dados experimentais (para refs. ver 7), a MEx não é uma doença muscular generalizada, restringindo-se apenas a um pequeno grupo de músculos, dependente do exercício realizado. A intensidade das lesões, bem como a percentagem de fibras atingidas pelas anomalias histológicas, estão dependentes de numerosos factores (para refs. ver $6,7,17$ ), tendo principal destaque: (i) a intensidade e a duração do exercício efectuado, (ii) o músculo observado, (iii) o tipo de fibra muscular analisada, (iv) o tempo que medeia entre a finalização do exercício e a recolha da amostra, (v) o nível de treino do indivíduo, (vi) o tipo de contracção predominantemente realizado, (vii) o modelo experimental utilizado, (viii) a idade do modelo experimental e (ix) o estado de inervação do músculo em causa.

A descrição das lesões histológicas associadas à MEx nem sempre é concordante de autor para autor em consequência não só dos factores condicionantes atrás mencionados, mas também das diferenças evidenciadas no horário de recolha das amostras e dos objectivos do trabalho (para refs. ver 6,7 ).

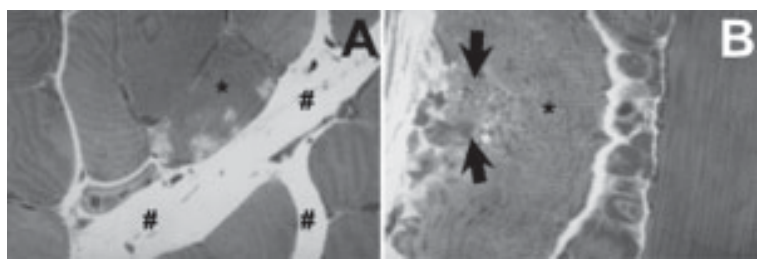

Figura 1. Fotografias de microscopia óptica de músculo gastrocnémius, em corte transversal (A, 393x) e longitudinal (B, 493x) de animal sacrificado 24 horas após exercício, mostrando um espaço intersticial alargado (\#) e a presença de fibras $\left({ }^{*}\right)$ com áreas de necrose segmentar (parcial). É visível, junto de uma área necrosada (setas), uma infiltração celular, provavelmente de fagócitos. As fibras adjacentes possuem uma estrutura normal.

De uma maneira simples, poderíamos definir as alterações morfológicas da MEx como lesões de carácter focal, sub-letais e letais (com posterior necrose), atingindo pequenas áreas de algumas das fibras recrutadas (Fig. 1) $(3,4,7,17)$.

Conciliando os dados experimentais $(8,9,16)$, facilmente se conclui que estas alterações, letais e 
sub-letais induzidas pelo exercício, são idênticas às descritas para a célula em geral, apresentando, no entanto, alterações histológicas adicionais que estão, naturalmente, relacionadas com a especialização da fibra muscular.

Um tipo de alterações, consequente da diferenciação da fibra muscular, é vulgarmente encontrado, em cortes longitudinais à microscopia de luz, logo após a finalização do exercício físico e consiste, estruturalmente, na desorganização do padrão estriado (Fig. 2 e 3 ).

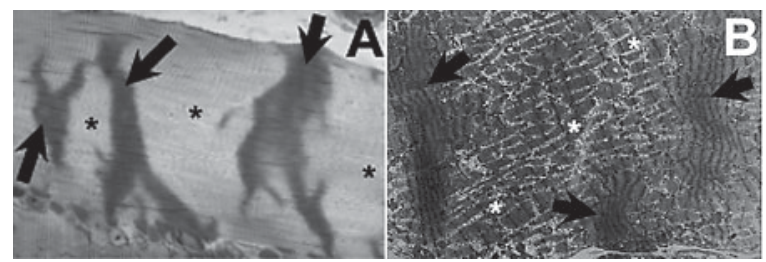

Figura 2. Fotografias de microscopia óptica (A, 615x) e electrónica (B. 918x) em corte longitudinal, de músculo soleus de animais sacrificados logo após exercício, mostrando a presença de irregularidades do padrão estriado $\left(^{*}\right)$ intervaladas com zonas de contracção das miofibrilas (setas).

Esta desorganização, habitualmente designada por irregularidade do padrão estriado, manifesta-se por um alargamento das bandas isotrópicas (I) e anisotrópicas (A), com esbatimento da coloração muscular, estando frequentemente intercalada por áreas onde a largura das bandas I se encontra diminuída, apesar das dimensões das bandas A permanecerem constantes, sugerindo interacção das proteínas contrácteis nessas zonas $(3,6,7)$. Em consequência, a coloração muscular nessas áreas é mais carregada (Fig. 2 e 3 ).

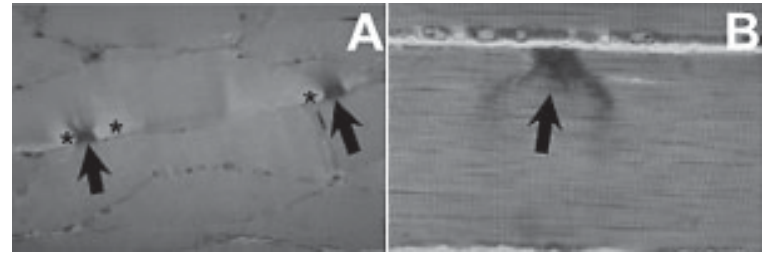

Figura 3. Fotografias de microscopia óptica, do músculo soleus, em corte longitudinal (A, 313x, B, 615x), de animais sacrificados logo após exercício, mostrando irregularidades do padrão estriado $\left(^{*}\right)$ intervaladas por zonas de contracção das miofibrilas (setas). De notar que estas zonas de interacção miofibrilar estão intimamente relacionadas com o sarcolema.
De acordo com a literatura $(6,7)$, estas alterações estruturais poderão ter uma origem mecânica, uma vez que (1) são observadas logo após a finalização do exercício físico, (2) parecem relacionar-se directamente com a percentagem de contracções excêntricas realizadas, (3) a sua incidência e intensidade não se modificam em função do tempo decorrido após exercício e (4) revelam uma coloração pálida, talvez pela diminuição de material contráctil induzida pela tracção mecânica.

Consequente às fortes tracções mecânicas a que as fibras se sujeitam, não só as proteínas contrácteis e do citosqueleto podem ficar afectadas, mas também as diferentes membranas celulares podem ser atingidas (Fig. 3), com a consequente perda da homeostasia celular a vários iões, particularmente ao ião cálcio (14).

Em termos ultra-estruturais, as irregularidades do padrão estriado, observadas à microscopia de luz, parecem corresponder às alterações nas linhas $Z$, tais como a disrupção, o esbatimento ou a extensão do material dessas linhas para as bandas I adjacentes (6, 7). Esta anomalia, atinge, frequentemente, a totalidade das linhas $\mathrm{Z}$ dos sarcómeros adjacentes, ocupando, frequentemente, toda a largura da fibra muscular. Em muitos casos, o número de sarcómeros em série atingidos ultrapassa as duas dezenas (6). À microscopia electrónica, as zonas de maior coloração que as intercalam resultam, de facto, da diminuição do comprimento dos sarcómeros, com acentuada redução das bandas I sem qualquer alteração nas dimensões das bandas A (Fig. 2). Quadro morfológico semelhante pode também ser encontrado em indivíduos saudáveis e assintomáticos, embora com menor incidência e intensidade (resultados não publicados). Estas zonas de maior densidade tecidual correspondem, necessariamente, a áreas onde as proteínas contrácteis interagem, sugerindo, por isso, a existência de grandes quantidades de ião cálcio sarcoplasmático disponível para ligação à troponina $C(7,14)$. Este ião poderá ter origem extra-celular, por atingimento sarcolemal, como a Fig. 3 sugere, ou intra-celular, por perda da permeabilidade selectiva da membrana do retículo sarcoplasmático (16). Logo após a finalização do exercício físico, particularmente se este tiver um predomínio de 
contracções concêntricas, um outro tipo de alteração estrutural, frequentemente observada à microscopia de luz, consiste na irregularidade de coloração ao ácido periódico de Schiff (PAS) verificada entre as diferentes fibras musculares.

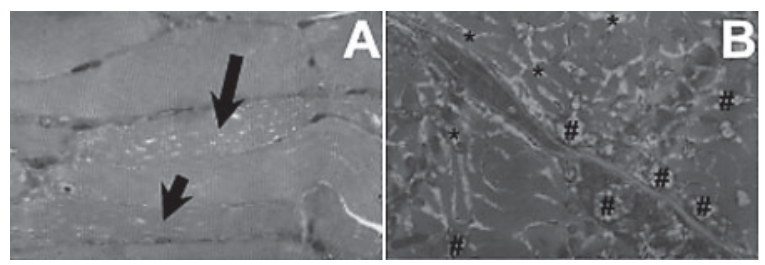

Figura 4. Fotografias de microscopia óptica, em corte longitudinal ( $A$ 393x), e electrónica (B, 1267x), em corte transversal, do músculo soleus, de animais sacrificados logo após exercício, mostrando fibras portadoras de vacuolização sarcoplasmática (setas), sem irregularidades do padrão estriado. Na fotografia B é de realçar o alargamento do espaço inter-miofibrilar, traduzindo edema intracelular $\left({ }^{*}\right)$, e o swelling mitocondrial (\#) bastante acentuado.

Nas fibras de tonalidade mais pálida, sugerindo um menor conteúdo em glicogénio e, por isso,

testemunhando o seu recrutamento durante o exercício, são também frequentemente observados inúmeros vacúolos sarcoplasmáticos (Fig. 4). Estas fibras, à análise ultra-estrutural, apresentam um maior espaço inter-miofibrilar bem como a presença de mitocôndrias edemaciadas ("swelling" mitocondrial), de matriz pálida e com irregularidades das suas cristas (Fig. 4).

Exceptuando a ligeira dilatação do retículo sarcoplasmático, verificada nas fibras portadoras das anomalias mitocondriais, os restantes organelos não apresentam, habitualmente, qualquer alteração digna de registo imediatamente após o exercício. Para além da deplecção de glicogénio, também o edema intracelular (Fig. 4 e 5) pode ser responsabilizado pela tonalidade mais pálida destas fibras. O edema intracelular pode ser perfeitamente justificado pela ineficácia da ATPase transportadora de $\mathrm{Na}^{+} / \mathrm{K}^{+}$, motivando uma concentração crescente de sódio no interior da fibra, arrastando consigo água do espaço intersticial. Simultaneamente, verifica-se uma saída de potássio com a consequente diminuição do $\mathrm{pH}$ intra-celular (para refs. ver 14).

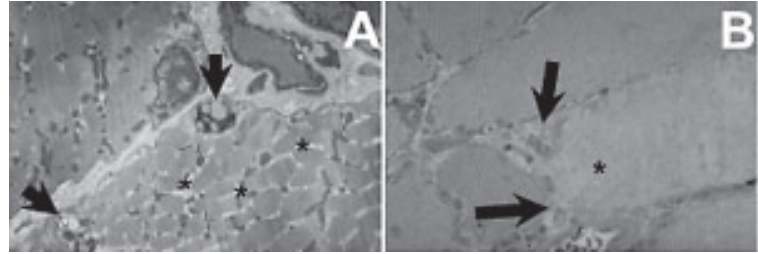

Figura 5. Fotografias de microscopia electrónica (A, 1339x), em corte transversal, e de microscopia óptica (B, 493x) em corte longitudinal. de um animal sacrificado 48 horas após exercício. Na fotografia A. na fibra inferior, é notório o edema intra-celular $\left(^{*}\right)$ e dois lisossomas secundários (setas). Na fotografia B é evidente uma zona com irregularidades do padrão estriado $\left(^{*}\right)$, onde se torna difícil identificar as bandas isotrópicas e anisotrópicas, e uma área de infiltração de fagócitos (setas).

A vacuolização sarcoplasmática parece ser, em grande parte, o resultado das anomalias mitocondriais observadas em microscopia electrónica. No entanto, é provável que a ligeira dilatação do retículo sarcoplasmático, consequente ao edema intra-celular, possa também contribuir, ainda que de forma menos intensa, para esse fenómeno (6). O facto destas anomalias serem observadas após exercícios com predomínio de contracções concêntricas, de atingirem a sua expressão máxima algumas horas após a finalização do exercício e de ocorrerem em fibras pálidas à coloração pelo PAS, sugere uma origem metabólica para estas alterações $(3,6,7,18,19)$. Uma hipotética explicação para a ocorrência destes fenómenos poderá estar relacionada com a perda celular da homeostasia ao ião cálcio $(7,15,16)$. Assim, consequente ao progressivo aumento da concentração sarcoplasmática do ião cálcio e à captação deste ião pelas mitocôndrias adjacentes, verifica-se uma redução da permeabilidade selectiva da membrana interna mitocondrial com o consequente edema (com atenuação do gradiente protónico) e desacoplação da fosforilação oxidativa (para refs. ver 14). Situação idêntica pode também ser motivada pela elevação térmica tecidual $(7,14)$. Esta poderia ser uma hipotética explicação para a ocorrência de vacuolização sarcoplasmática em fibras depleccionadas em glicogénio mas não portadoras de áreas com interacção das suas proteínas contrácteis (Fig. 4). 
Um outro tipo de anomalias, também relacionado com a diferenciação das fibras e que difere ligeiramente da descrição feita para a célula em geral, tem a ver com o facto da fibra muscular ser um sincício morfológico que, quando sujeito a um estímulo lesivo letal, não degenera de uma forma integral mas sim parcial (Fig. 1 e 6), ficando os núcleos das áreas não atingidas a assegurar o funcionamento dessas zonas (7).

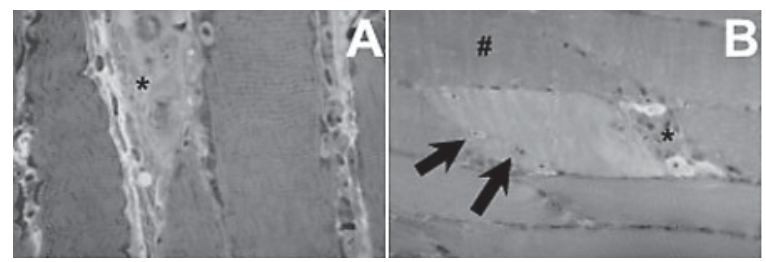

Figura 6. Fotografias de microscopia óptica, do músculo soleus em corte longitudinal (A, 493x; B, 493x), de animais sacrificados 48 horas após exercício, com a presença de zonas de necrose segmentar $\left({ }^{*}\right)$ infiltradas por fagócitos. É ainda observada uma fibra com alguma vacuolização (\#) e núcleos centrais (setas).

O aparecimento destas áreas de necrose segmentar só se verifica, normalmente, algumas horas após o término do exercício. De facto, um a dois dias após o exercício físico, todo o quadro anatomopatológico se torna mais exuberante, caracterizando a natureza retardada da MEx.

Durante este período, as irregularidades do padrão estriado continuam a ser observadas, diferindo estruturalmente, contudo, daquelas observadas logo após a finalização do exercício. Há uma menor definição da estriação com a consequente dificuldade ou mesmo impossibilidade de identificação das bandas A e I (Fig. 5).

Tudo indica que haja uma substituição, progressiva, das características das irregularidades do padrão estriado ao longo do tempo pós-exercício (6). Esta suposição parece apoiar a hipótese de que a disrupção miofibrilar, verificada durante a realização do exercício físico, pode expor os seus componentes estruturais à acção de proteases neutras ou alcalinas que se encontram no espaço inter-miofibrilar, provavelmente associadas ao retículo sarcoplasmático $(6,7,15)$ agravando, desta forma, as lesões preexistentes. É possível que as elevadas concentrações sarcoplasmáticas de ião cálcio possam contribuir para a activação destas enzimas (para refs. ver 7 ).

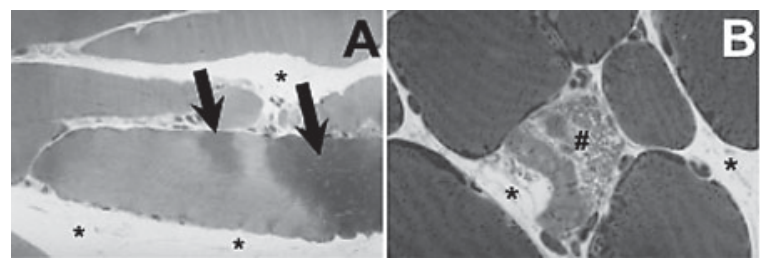

Figura 7. Fotografias de microscopia óptica, em corte longitudinal ( $A$, 393x) e transversal (B, 493x) do músculo soleus, de animais sacrificados 48 horas após exercício, mostrando edema intersticial $\left(^{*}\right)$ e zonas com desaparecimento das bandas I e vacuolização simultânea (setas). É ainda possível observar-se uma fibra em necrose de coagulação ladeada por fibras normais (\#).

Zonas com redução ou desaparecimento total das bandas I continuam a ser observadas muitas horas após a finalização do exercício e, particularmente nestas áreas, a vacuolização sarcoplasmática é perfeitamente identificável (Fig. 7). Esta constatação vem reforçar a hipótese da perda da homeostasia celular ao cálcio como uma das possíveis etiologias para as alterações encontradas $(15,16)$.

Nas fibras portadoras de alterações estruturais, à análise por microscopia electrónica, é notório, com o aumento do tempo pós-exercício, o progressivo incremento do volume mitocondrial (com irregularidades da matriz e das cristas), mais proeminente nas zonas sub-sarcolemais, embora também observado nas regiões inter-miofibrilares (6), assim como a existência de mitocôndrias, intercaladas, com aspecto normal (Fig. 4 e 5). O espaço inter-miofibrilar mantém-se aumentado (Fig. 5) e as anomalias das linhas $Z$, do retículo sarcoplasmático e do comprimento dos sarcómeros são também observadas com mais evidência. Nestas fases pós-exercício é possível detectar-se um aumento dos complexos de Golgi, dos corpos residuais, dos vacúolos autofágicos e dos lisossomas primários e/ou secundários (Fig. 5). Estes são, sem dúvida, sinais morfológicos reveladores de uma actividade autofágica aumentada $(6,7)$. Estas evidências são principalmente visíveis no espaço subsarcolemal das fibras que circundam as áreas lesadas e principalmente nas fibras com elevado conteúdo 
mitocondrial (para refs. ver 6). Estes sinais histológicos de autofagia, nomeadamente os vacúolos autofágicos e os corpos esfero-membranosos, são pouco frequentes na primeira hora após a finalização do exercício, aparecendo com maior frequência entre o $2^{\circ}$ e o $7^{\circ}$ dia (para refs. ver 6 ).

Em termos gerais, tudo indica que ao longo do tempo após exercício, as fibras agredidas iniciam um processo de autodestruição, lisando as miofibrilas e promovendo não só a disrupção das mitocôndrias, do sarcolema e do retículo sarcoplasmático, mas também a autofagocitose e a activação lisossómica $(6,7)$. As anomalias histológicas musculares, motivadas por este processo intrínseco às próprias fibras, apesar de apresentarem algumas características próprias, relacionadas com a diferenciação celular, constituem um bom exemplo da acentuada analogia morfológica verificada na resposta dos diferentes tecidos à agressão em geral $(3,17)$. O facto da resposta morfológica muscular induzida pelos diferentes tipos de estímulos lesivos ser também análoga $(1,2,8,10$, $11,19,21)$, reforça ainda mais a hipótese dos mecanismos bioquímicos de degradação celular serem comuns às diferentes agressões.

Entre 4 a 6 horas pós-exercício, é vulgar observar-se a infiltração, em grande quantidade, de mononucleares, bem como de alguns polimorfonucleares, com carácter focal, bem evidente na periferia das áreas necrosadas (Fig. 5, 6, 8,9 e 10).

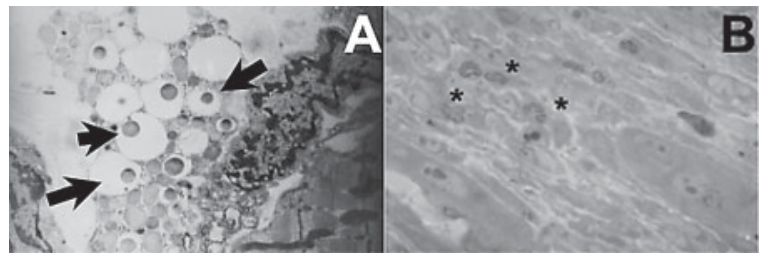

Figura 8. Fotografias de microscopia electrónica (A, 1540x) e microscopia óptica (B, 615x), do músculo soleus, de animais sacrificados 24 horas após exercício. É notória uma célula com vacúolos típicos (setas) das "Natural Killer Cell", e uma área de infiltração de mononucleares e polimorfonucleares $\left({ }^{*}\right)$ onde também são visíveis algumas estruturas celulares. hipoteticamente mioblastos e miotúbulos.
Um a três dias após o exercício, a resposta inflamatória nos músculos agredidos parece estar completamente estabelecida, com fagócitos dispersos pelo endomísio e no interior de algumas fibras lesadas (Fig. 8, 9 e 10) (9). Os mononucleares incluem principalmente macrófagos, originados dos monócitos que infiltraram o músculo, e alguns linfócitos B, T e linfócitos T citotóxicos (Fig. 8, 9 e 10 ), originando um infiltrado celular semelhante ao descrito nas miopatias inflamatórias, apesar dessa invasão linfocitária, motivada pelo exercício, nem sempre ser descrita na literatura (para refs. ver 7). O facto das fibras agredidas poderem expor determinados antigéneos, até aí estranhos ao sistema imunológico, poderá justificar a activação e participação linfocitária tanto nas miopatias inflamatórias como nas lesões consequentes ao exercício (7). A presença de grandes quantidades de monócitos também poderá ser explicada pelo facto dos seus factores quimiotácticos específicos serem, aparentemente, os produtos de degradação do colagénio, cujo aparecimento se verifica logo após o exercício (para refs. ver 6,7 ). A presença de alguns eosinófilos (Fig. 9) poderia sugerir uma etiologia imunológica, hipótese até agora não confirmada.

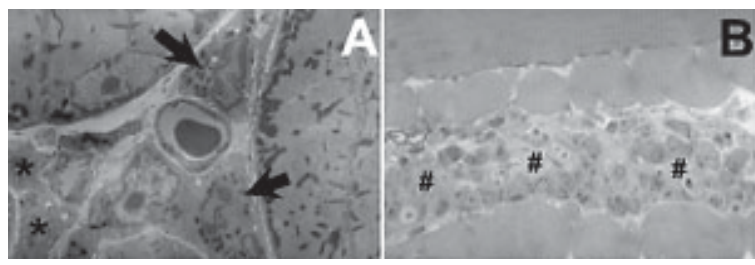

Figura 9. Fotografias de microscopia electrónica (A, 1125x) e microscopia óptica (B, 493x), do músculo soleus, de animais sacrificados 48 horas após exercício, onde são identificados dois eosinófilos (setas), um fibroblasto em actividade $\left(^{*}\right)$ e uma área de infiltração leucocitária (\#).

Com excepção do tipo predominante dos leucócitos invasores, a resposta inflamatória aguda, motivada pela agressão do exercício físico, parece ser idêntica ao modelo padrão, estando descritos desgranulação mastocitária, elevação do catabolismo muscular, aumento da permeabilidade vascular com edema tecidual e presença de factores do complemento, de fibrinogénio e de albumina nas zonas lesadas (para refs. ver 6,7$)$. Atendendo ao potencial efeito 
citotóxico das substâncias libertadas pelos leucócitos, bem como ao seu carácter não selectivo (9), coloca-se hoje a hipótese que os leucócitos possam contribuir para o exagero, não só das anormalidades bioquímicas tradutoras de agressão oxidativa, mas também das alterações histológicas descritas, tal como o observado em situações de isquemia/reperfusão muscular esquelética e cardíaca (para refs. ver. 7). Esta hipótese é reforçada pela aparente atenuação das lesões musculares, induzidas pela actividade física, exercida por determinados fármacos com actividade anti-inflamatória (9).

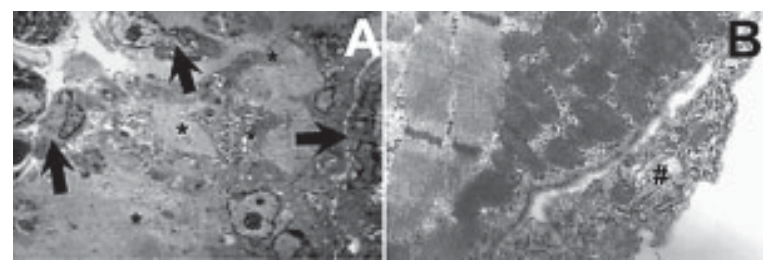

Figura 10. Fotografias de microscopia electrónica de animais sacrificados 24 horas após (A, 1339x) e 96 horas após exercício (B. 2108x). É visível uma área necrosada (*) infiltrada com fagócitos (setas) e um fibroblasto em actividade(\#).

Algumas horas após o exercício, é usual o aparecimento de núcleos em posição central nas áreas lesadas (Fig. 6 e 11), bem como nas zonas adjacentes a estas $(8,9,16)$. Núcleos em posição central, são um sinal inequívoco de imaturidade muscular e, portanto, de áreas em regeneração ou de novas fibras em crescimento (para refs. ver 6, 7). Estes núcleos provêm da estimulação e da consequente diferenciação das células satélite em mioblastos com posterior fusão à fibra adjacente. A ocorrência destes núcleos em posição central constitui, por isso, um bom indicador da existência prévia de lesão muscular. Contudo, é importante frisar que indivíduos saudáveis, sem história de actividade física recente, podem apresentar núcleos centrais até $2 \%$ do total de fibras observadas (para refs. ver 6,7$)$. De facto, no dia-a-dia, pelas agressões a que as fibras são sujeitas durante o seu normal recrutamento, existe uma contínua activação de células satélite (apesar de possuir uma expressão limitada), motivando a contínua presença de núcleos centrais em percentagem reduzida.

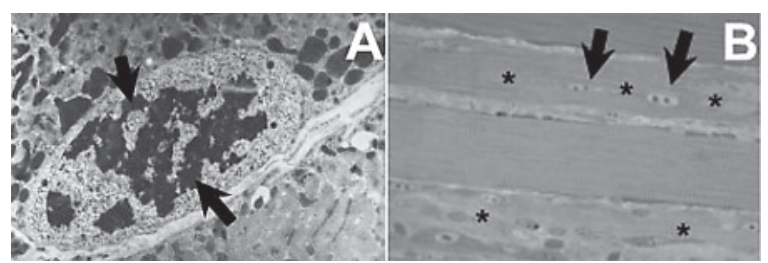

Figura 11. Fotografias de microscopia electrónica (A, 1339x), de músculo soleus de animal sacrificado 24 horas após exercício, e de microscopia óptica $(B, 393 x)$ do músculo gastrocnémius de um animal sacrificado 1 semana após exercício. É visível uma célula satélite activada, em fase $M$, com cromossomas em formação (setas). Podem ser observados núcleos em posição central (setas) e zonas de regeneração onde a estriação ainda não se encontra perfeitamente organizada $\left(^{*}\right)$

Actualmente, pensa-se que estas lesões musculares associadas à MEx não resultem exclusivamente das contracções excêntricas, como até há alguns anos se defendia (para refs. ver 6, 7). Como atrás ficou expresso, tudo indica que o quadro anatomopatológico possa ser inconstante, apresentando um carácter variável, com maior frequência de um ou outro tipo de alterações estruturais, consoante o predomínio da sobrecarga mecânica ou metabólica do exercício efectuado. Parece-nos, pois, provável a existência de vários padrões de anomalias estruturais musculares associadas à MEx, com etiologias aparentemente diferentes $(3,6,7,18,19)$, apesar de possuírem uma fisiopatologia semelhante e, de alguma forma, relacionada com a perda da homeostasia celular ao cálcio. Independentemente da etiologia e da fisiopatologia, duas a três semanas após o exercício já a estrutura do tecido adquiriu um aspecto normal $(6,7)$.

\section{CORRESPONDÊNCIA}

\section{José Alberto Ramos Duarte}

Gabinete de Biologia do Desporto

Faculdade de Ciências do Desporto

e de Educação Física

Universidade do Porto

Rua Dr. Plácido Costa, 91

4200.450 Porto

Tel: +351225074700

[jarduarte@fcdef.up.pt] 


\section{REFERÊNCIAS}

1. Appell H-J, Duarte JA, Glöser S, Remião F, Carvalho F, Bastos ML, Soares JM (1997). Administration of tourniquet: II. Prevention from postischemic oxidative stress can reduce muscle edema. Arch. Orthop. Trauma. Surg. 116: 101-105. 2. Appell H-J, Glöser S, Duarte JA, Zellner A, Soares JMC (1993). Skeletal muscle damage during tourniquet-induced ischemia. The initial step towards atrophy after orthopaedic surgery? Eur. J. Appl. Physiol. 67: 342-347.

3. Appell H-J, Soares JMC, Duarte JA (1992). Exercise, muscle damage and fatigue. Sports Med. 13: 108-115.

4. Appell H-J, Soares JMC, Duarte JA (1999). O exagero da agressão muscular esquelética induzida pelo exercício. In: Educação Física: Contexto e Inovação. I Vol. A. Marques, A. Prista, A.F. Júnior (Eds). Faculdade de Ciências do Desporto e de Educação Física da Universidade do Porto e Faculdade de Ciências da Educação Física e do Desporto Universidade Pedagógica de Maputo, pp: 139-144.

5. Carvalho J, Duarte JA, Soares JMC (1993). Miopatia do exercício. Breve revisão da literatura. Horizonte X(57): 95-100. 6. Duarte JA (1989). Miopatia do Exercício - Alterações Estruturais no Músculo Esquelético do Ratinho Sujeito a Dois Protocolos Diferentes de Corrida. Provas de Aptidão Pedagógica e Capacidade Científica, Faculdade de Ciências do Desporto e de Educação Física, Universidade do Porto.

7. Duarte JA (1993). Lesões Celulares do Músculo Esquelético Induzidas pelo Exercício. Tese de Doutoramento em Ciências do Desporto, Faculdade de Ciências do Desporto e de Educação Física, Universidade do Porto.

8. Duarte JA, Carvalho F, Bastos ML, Soares JMC, Appell H-J (1993). Endothelium-derived oxidative stress may contribute to exercise-induced muscle damage. Int. J. Sports Med. 14: 440-443. 9. Duarte JA, Carvalho F, Bastos ML, Soares JMC, Appell H-J (1994). Do invading leucocytes contribute to the decrease in glutathione concentrations indicating oxidative stress in exercised muscle, or are they important for its recovery? Eur. J. Appl. Physiol. 68:48-53.

10. Duarte JA, Carvalho F, Natsis K, Bastos ML, Soares JMC, Appell H-J (1999). Structural alterations of skeletal muscle induced by chronic administration of d-amphetamine and food restriction Basic \& Appl. Myology. 9: 65-69.

11. Duarte JA, Carvalho F, Natsis K, Remião F, Bastos ML, Appell H-J, Soares JMC (1999). Repeated administration of damphetamine induces structural alterations in skeletal muscle. Med. Sci. Sports \& Exerc. 31: S166.

12. Duarte JA, Magalhães JF, Monteiro L, Almeida-Dias A, Soares JMC, Appell H-J (1999). Exercise-induced signs of muscle overuse in children. Int. J. Sports Med. 20: 103-108. 13. Duarte JA, Soares JMC (1990). Sensação retardada de desconforto muscular. Etiologia, fisiopatologia, tratamento e prevenção. Rev. Port. Med. Desp. 8: 155-162.

14. Duarte JA, Soares JMC (1991): Etiologia da fadiga muscular. Alguns factores condicionantes. Rev. Port. Med. Desp. 9: $165-174$

15. Duarte JA, Soares JMC (1991). Contribuição do ião cálcio na miopatia do exercício. In: As Ciências do Desporto e a Prática Desportiva, Vol. 2. Eds: J. Bento e A. Marques. Faculdade de Ciências do Desporto e de Educação Física da Universidade do Porto, pp: 95-101.

16. Duarte JA, Soares JMC, Appell H-J (1992). Nifedipine diminishes exercise-induced muscle damage in mouse. Int. J. Sports Med. 13: 274-277.
17. Soares JMC, Duarte JA (1989). Miopatia do exercício. Etiologia, fisiopatologia e factores de prevenção. Rev. Port. Med. Desp. 7: 187-200.

18. Soares JMC, Duarte JA, Appell H-J (1992). Exerciseinduced muscle damage. Mechanic and/or metabolic origin. Congress Book of Eleventh Congress of Sports Medicine of the A.Z. ST-Jan, Bruge, Belgium, pp: 73-77.

19. Soares JMC, Duarte JA, Appell H-J (1992). Metabolic vs. mechanical origin of muscle lesions induced by exercise. Int. J. Sports Med. 13: 84.

20. Soares JMC, Duarte JA, Appell H-J (1993). Is serum CK related to muscular fatigue in long-distance running? In: Neuromuscular Fatigue. A.J. Sargeant \& D. Kernell (Eds.), Academy Series. Royal Netherlands Academy of Arts and Sciences - North Holland, Amsterdan, pp: 76-78.

21. Soares JMC, Duarte JA, Carvalho J, Appell H-J (1993). The possible role of intracellular $\mathrm{Ca}^{2+}$ accumulation for the development of immobilization atrophy. Int. J. Sports Med. 14: 437-439

22. Soares JMC, Mota P, Duarte JA, Appell H-J (1996). Children are less susceptive to exercise-induced muscle damage than adults - a preliminary investigation. Pediatr. Exerc. Sci. 8: 361-367. 